\title{
Comparison of photopolymers for optical data storage applications and relief diffractive optical elements recorded onto photopolymers
}

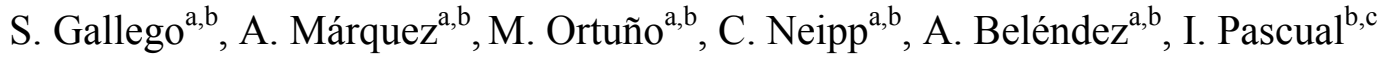 \\ ${ }^{a}$ Dept. Física Enginyeria de Sistemes i Teoria del Senyal, Universitat d'Alacant \\ (Spain) Apartat 99 E-03080 Alacant \\ ${ }^{\mathrm{b}}$ I.U. Fisica Aplicada A Las Ciencias Y Las Tecnologías (Spain) Apartat 99 E-03080 \\ Alacant \\ ${ }^{\mathrm{c}}$ Dept. Óptica, Farmacologia i Anatomia, Universitat d'Alacant (Spain) Apartat 99 E- \\ 03080 Alacant
}

\begin{abstract}
In this paper four different photopolymers are compared on the basis of their suitability for holographic data storage. The optical recording parameters of these photopolymers were directly determined using the zero spatial frequency limit. The behavior of cover plated and uncover plated material layers was analyzed. Once the main parameters were determined, we proposed a novel model to simulate the recording of relief diffractive elements onto the photopolymers without cover plating. Relief surface changes provide interesting possibilities for storing diffractive optical elements on photopolymers and are an important source of information for characterizing and understanding the material behaviour. In this paper we also present a 3-dimensional model, based on direct parameter measurements, for predicting the relief structures generated on the material. This model was successfully applied to different photopolymers with different values of monomer diffusion.
\end{abstract}

Keywords: Holography, Holographic recording materials, diffractive optics

\section{INTRODUCTION}

Photopolymer materials are practical materials for use as holographic recording media since they are inexpensive, self-processing, and have the ability to record low loss, highly diffraction efficient, volume holographic gratings. In general these materials absorb light of the appropriate wavelength, causing photo-polymerization of the local monomer, inducing a change in the material's refractive index and thickness. Photopolymers have been widely studied in the range of holographic spatial frequencies (over 500 lines $/ \mathrm{mm}$ ). The potential of these materials for use in holographic applications has been demonstrated with great success [1-3]. Their wide-spread application [3-9] has made the use of photopolymers as optical materials an interesting field of research. They are useful for different applications, such as diffractive and refractive optical elements, due to the refractive index variations and relief profiles generated [4,5,7,9]. A photopolymer is basically made up of one or several monomers and crosslinkers, a sensitizer, binder and initiator. The composition of photopolymers may vary greatly and the recording layer can be manufactured in a wide range of possible thicknesses. These properties make them an interesting material for generating phase diffractive optical elements. Recently the capacity of photopolymers to record diffractive elements has been demonstrated [9].

Smart Nano-Micro Materials and Devices, edited by Saulius Juodkazis, Min Gu, Proc. of SPIE Vol. 8204, 820421 - (c) 2011 SPIE · CCC code: 0277-786X/11/\$18 - doi: 10.1117/12.902694 


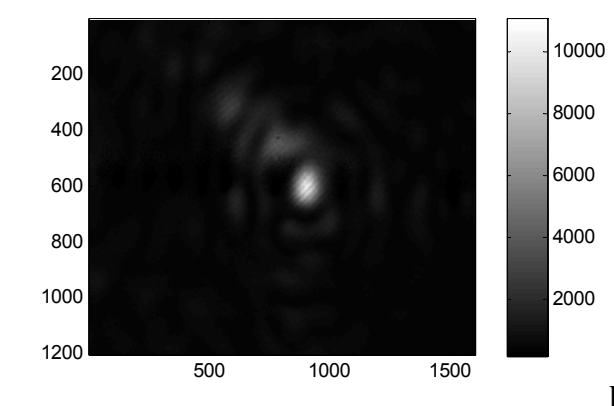

a)

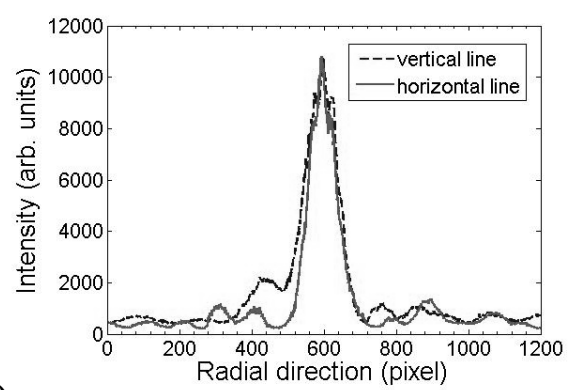

b)

Figure 1. In (a), defocused planes of the best image plane obtained at a distance $\mathrm{z}=68 \mathrm{~cm}$ from the plane of the diffractive phase lens recorded. In (b) we show the intensity profile along the horizontal and vertical lines passing through the peak intensity point in the best image plane.

In addition, photopolymers are often used as the base of holographic memory displays and great efforts have been made to model the photopolymers' behaviour for data storage applications [10]. Depending on the application, different photopolymer compositions are used. For each composition the main parameters: monomer diffusion, shrinkage, polymerization velocity, etc. should be determined previously.

This paper is divided into two parts. Initially the four different photopolymers are compared. The optical recording parameters were directly determined using the zero spatial frequency limit. The behavior of cover plated and uncover plated material layers was analyzed. Frequently in the literature, PVA materials, as opposed to commercial materials, are not cover plated [1]. Cover plating can provide mechanical support and alter surface tension (energy) effects so as to change the profile of the surface relief grating formed during exposure [11-13]. In the absence of cover plating, the layer may be more affected by the environment. For example, water may be absorbed or evaporate and the surface profile formed during exposure may be greatly changed. Furthermore, photopolymers without cover plating allow surface profiles and reflection diffractive elements to be generated, and provide new interesting information about the processes that take place in the materials during and after light exposure. Nevertheless, classically PVA materials are prepared with acrylamide as the main monomer, and this compound has an important drawback - it is carcinogenic. Therefore, we substituted acrylamide by sodium acrylate with good results [14]. Furthermore, we compared two cover plated materials - our PEA photopolymer (with and without liquid crystal molecules) - with a commercial layer optimized for data storage applications. The potential of PEA for producing dynamic diffractive elements was demonstrated.

After determining the main parameters, in the second part of this paper a novel 3-dimensional model to simulate the recording of relief diffractive elements onto the photopolymers without cover plating is described. Relief surface changes provide interesting possibilities for storing diffractive optical elements on photopolymers and are an important source of information for characterizing and understanding the material behaviour. The model based on direct parameter measurements can predict the relief structures generated on the material. This model was successfully applied to different photopolymers with different values of monomer diffusion and different spatial frequencies. The goal of this study is to determine the utility of a 3-D diffusion model for predicting the surface formation and evolution after exposure in order to make the theoretical predictions as accurate as possible. Once the model has been experimentally validated, some different values for monomer diffusion are provided and analyzed in order to evaluate the importance of this parameter in the manufacture of diffractive optical elements. Shrinkage caused by polymerization is greatly reduced after exposure due to monomer diffusion from the non-exposed to exposed zones [15-18]. In some cases this process produces swelling of the polymerized regions and the diffraction efficiency of the zero order rises to a maximum again. In this post-exposure process, polymerization does not take place and only species diffusion can modify the surface profile. We simulated different material behaviors and compared different materials in order to clarify the phenomenon of grating evolution in the dark. 


\section{PHOTOPOLYMERS CHARACTERIZED AND EXPERIMENTAL SET-UP}

In this section we briefly describe the four different materials compared. The first is PVA/acrylamide (PVA/AA) material (Photopolymer 1). Some variations of this material have been widely studied in the literature with good results [5,9]. However, since recent investigations confirm the toxic potential of AA [19], in the second material analyzed we substituted AA monomer by sodium acrylate (NaAO)[14] (Photopolymer 2). Both Photopolymer 1 and Photopolymer 2 have a thickness of around $100 \mu \mathrm{m}$. These two materials are analyzed without cover plating; nevertheless, the first attempts to use a PVA material with cover plating in order to avoid formation of relief gratings have been recently reported [13]. The third polymer is a PEA photopolymer (Photopolymer 3). This photopolymer is composed of dipentaerythritol penta/hexa-acrylate (DPHPA) as monomer and binder, N-vinyl pirrolidone (NVP) as crosslinker, ethyl eosin (YE) as dye and N-methyl diethanolamine (NMDETA) as radical generator. These components were mixed under red light where the material is not sensitive. The solution $(100 \mathrm{~mL})$ was sonicated in an ultrasonic bath, heated, mixed with a magnetic mixer and deposited between two glass plates $2 \mathrm{~mm}$ thick. A spacing of $50(\mu \mathrm{m})$ was obtained with spacers. This material is liable to contain liquid crystal particles, thus dynamic holograms or diffractive elements can be manufactured. We compared the behavior of this material with the Bayfol@ HX TP photopolymer (Photopolymer 4), one of the commercial photopolymers produced by Bayer MaterialScience. The thickness of the photopolymer is around $50 \mu \mathrm{m}$. In principle Bayer photopolymers are manufactured for holographic data storage and have been successfully tested for transmission and reflection geometries [1].

The experimental set-up is similar to the one used in ref. [14] to measure the phase-shift as a function of exposure energy. The setup has two arms with an angular separation of $15^{\circ}$, one to expose the recording material and the other, which is the interferometer, to measure, in real-time, the phase-shift. The recording material is perpendicularly oriented with respect to the interferometer axis in order to facilitate analysis of the interferometric results: at an oblique incidence both the Fresnel coefficients at the interface and the increase in distance in propagation across the layer should be taken into account. For the first three compositions we used a $532 \mathrm{~nm}$ recording beam and $633 \mathrm{~nm}$ beam to measure the phaseshift (these compositions have Yellowish Eosin as the dye and at this wavelength the dye is not absorbed).The Bayfol ${ }^{\circledR}$ HX TP photopolymer was exposed to a He-Ne laser (633nm) and we measured the phase modulation between exposed and non-exposed zones with a $453 \mathrm{~nm}$ laser, where the dye is less sensitive, using a very low intensity. The recording intensity was $0.5 \mathrm{~W} / \mathrm{cm}^{2}$ in all cases.

In order to record phase diffractive gratings we introduced a spatial light modulator (SLM) working in the amplitude only mode [15] to modulate the green beam.

\section{RESULTS AND DISCUSSION}

\subsection{Experimental comparison at zero spatial frequency limit}

In this section we experimentally compare the four different materials at the zero spatial frequency limit when storing a phase grating with a period of $168 \mu \mathrm{m}$. Firstly we compared photopolymer 1 (PVA/AA) with photopolymer 2 (PVA/NaO), both in transmission and reflection. As can be seen in Figure 2 similar results were obtained with layers around $50 \mu \mathrm{m}$ thick. 


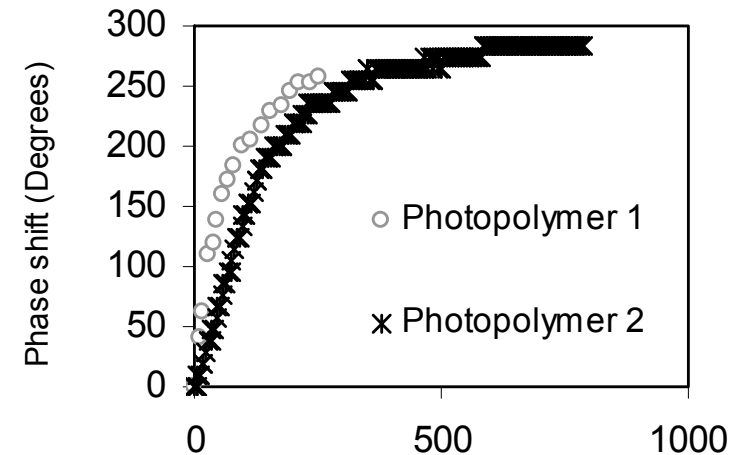

A)
Exposure time (s)

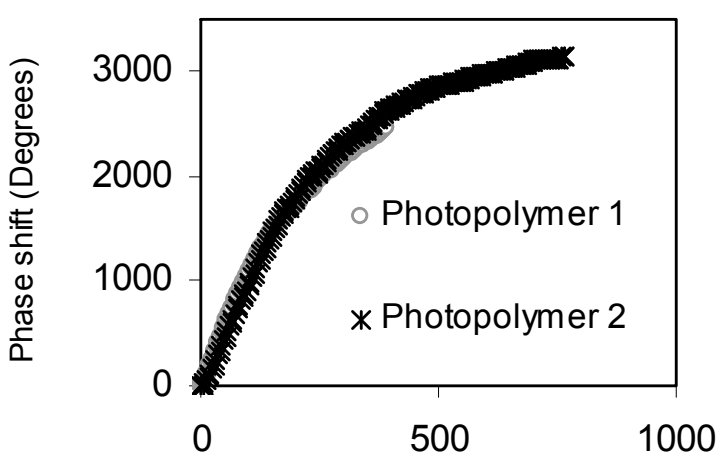

B)
Exposure time (s)

Figure. 2.- Behavior of Photopolymer 1 and Photopolymer 2 at the zero spatial frequency limit A) in transmission, B) in reflection.

With photopolymer 3 (PEA) and Photopolymer 4 (Bayfol) there is no shrinkage since they are both cover plated. Photopolymer 3 has a glass substrate as the cover plate and photopolymer 4 a plastic; thus measurements in reflection make no sense. The measurements in transmission are shown in Figure 3. As can be seen in this figure, the phase shift values are lower in cover plated photopolymers due to the invariance of thickness in the illuminated areas.

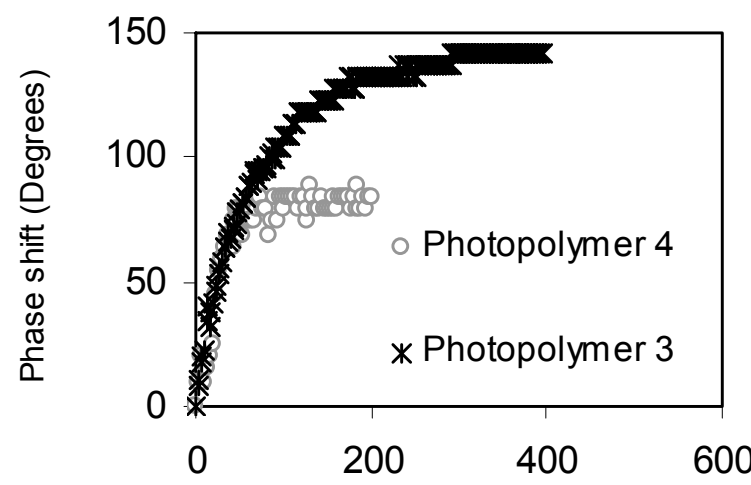

Exposure time (s)

Figure. 3. Behavior of Photopolymer 3 and Photopolymer 4 at the zero spatial frequency limit in transmission.

For these materials with cover plating we recorded gratings with periods of $168 \mu \mathrm{m}$ and $336 \mu \mathrm{m}$. The diffraction efficiency of the different orders was lower than $1 \%$ for photopolymer 4 , and for photopolymer 3 an efficiency of around $30 \%$ was achieved for orders \pm 1 . This different behavior can be explained by the difference in monomer diffusion in the two materials. We believe that monomer diffusion is clearly greater in PEA photopolymers.

\subsection{Theoretical model for predicting the relief structures formed in photopolymers without cover plating}

The results obtained at the zero spatial frequency limit for very low spatial frequencies help researchers to directly determine the main parameters of photopolymer behavior, such as polymerization rate, shrinkage, monomer diffusion, 
etc. [14]. Taking these values into account, we designed a surface model to predict the relief structures formed in photopolymers without cover plating. The model is based on the diffusion equations used in standard holographic models as can be seen in ref. [20]. The predictions for the surface structures provided by this model were confirmed by the experimental results obtained in the recording of diffractive gratings for photopolymers without cover plating. In particular, gratings with a spatial period of $168 \mu \mathrm{m}$ were recorded using a spatial light modulator [15]. The good agreement between the values for the four main diffracted orders predicted by the model and the experimental results shows the importance of the real zero frequency characterization in order to understand the material behavior. To check the values of monomer diffusion $\left(D_{m}\right)$ obtained in a previous analysis [15] we recorded for only a few seconds and observed how the diffracted order evolved after recording. At this stage polymerization has stopped and only monomer diffusion takes place. Therefore, shrinkage produced by polymerization is reduced due to the transfer of monomer from dark to bright zones. The results are shown in Fig. 4 for photopolymers 1 and 2. The gratings were recorded for a while (16 s for photopolymer 1 , and $30 \mathrm{~s}$ for photopolymer 2 ) and after recording, the evolution of the diffracted orders was measured in order to analyze the monomer diffusion effects. Using these simulations we accurately fitted the value of $D_{m}$ for PVA/AA materials $\left(1.510^{-8} \mathrm{~cm}^{2} / \mathrm{s}\right)$ and for PVA/NaAO $\left(1.210^{-9} \mathrm{~cm}^{2} / \mathrm{s}\right)$.

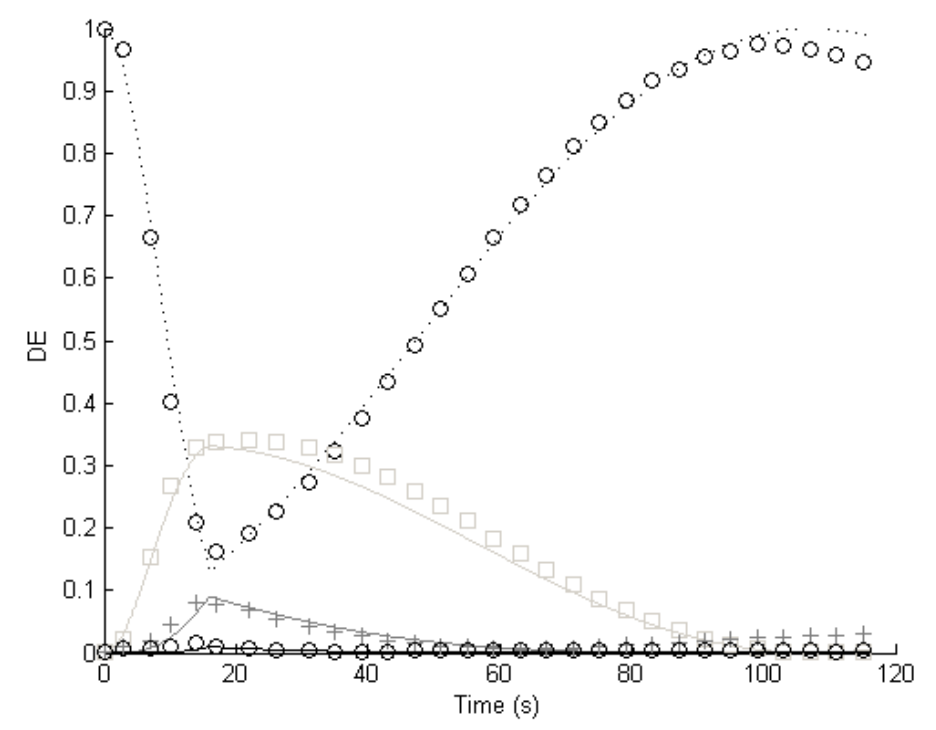

A) 


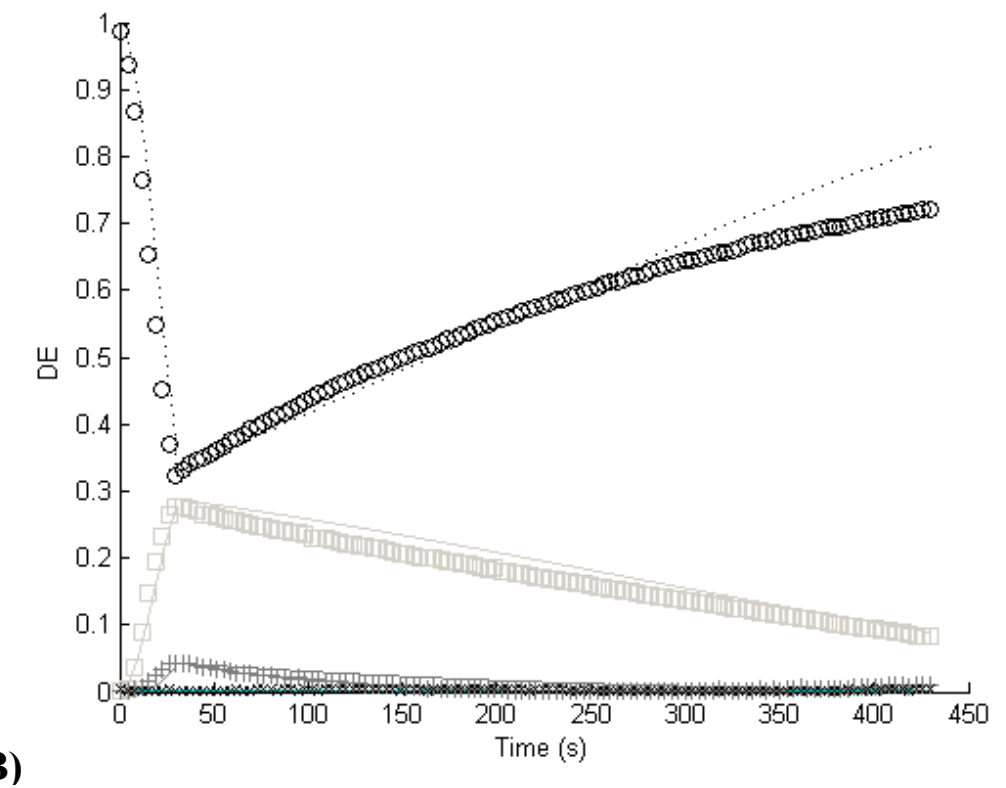

Fig. 4. Simulated diffraction efficiency for order 0 (circles), 1 (squares), 2 (gray cross), 3 (black cross) and experimental dots as a function of time with diffusion in depth. A) PVA/AA. B)PVA/NaAO.

Once monomer diffusion has been demonstrated, it is interesting to verify the reproducibility of the model for long recording times with these two photopolymer types. For long exposures, it was experimentally shown that the energy of the first four orders rises less than $10 \%$ due the high phase depth values [15]. The agreement between simulation and experiments can be deduced from Figure 5. The simulation and experiments were done using PVA/AA materials 100 $\mu \mathrm{m}$ thick, and the other parameters introduced were taken from the "zero spatial frequency" analysis $\left(\mathrm{D}=1.510^{-8} \mathrm{~cm}^{2} / \mathrm{s}\right.$; $\mathrm{K}_{\mathrm{R}}=0.007 \mathrm{~cm}^{2} /(\mathrm{s} \cdot \mathrm{mW})$ [20], (shrinkage) $\mathrm{Sh}=4 \mu \mathrm{m}[20], \alpha=0.02 \mu \mathrm{m}^{-1}[20], \mathrm{M}_{0}=0.22[20], \mathrm{I}=0.5 \mathrm{~mW} / \mathrm{cm}^{2}, \gamma=1$. 
A)
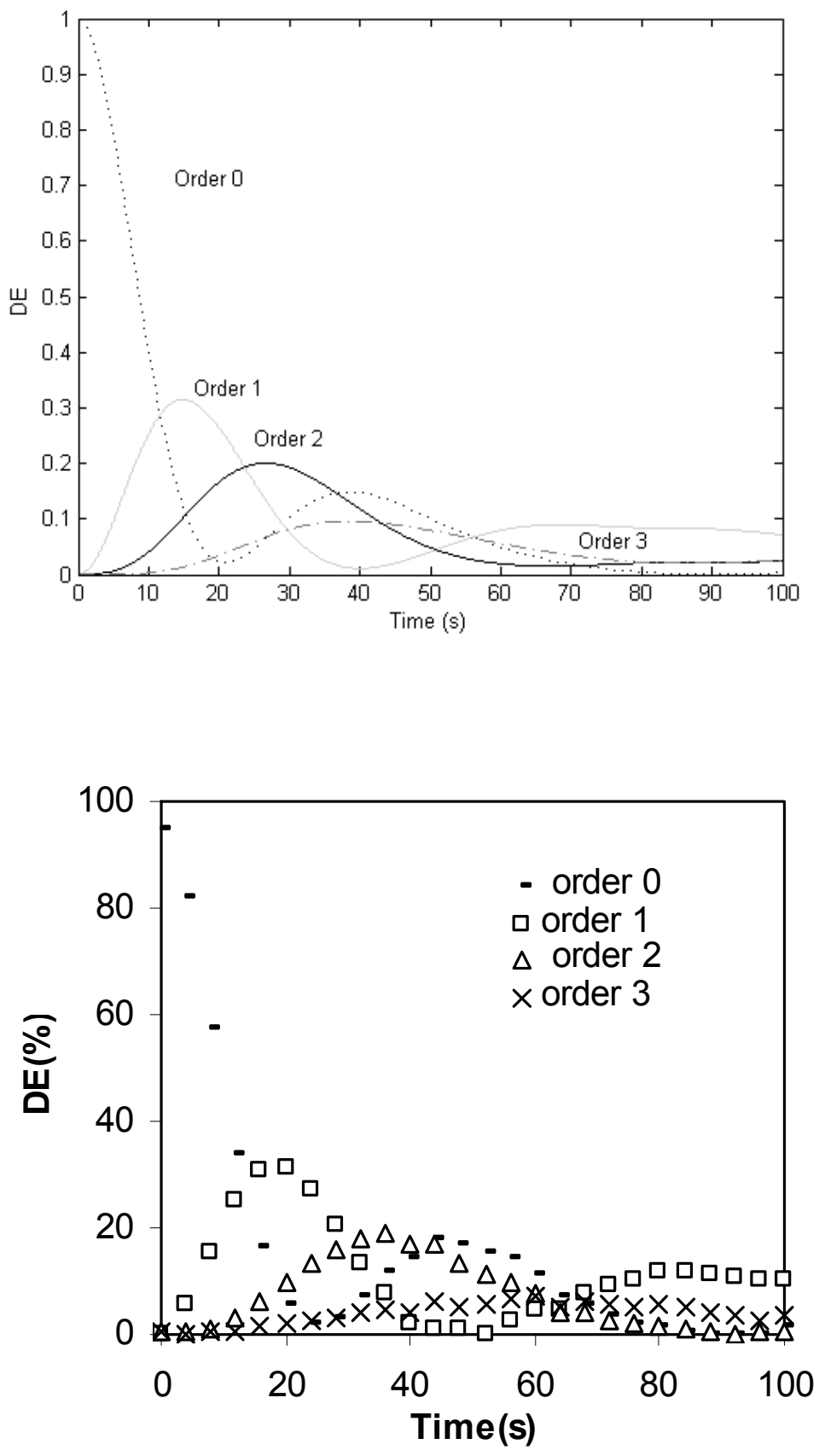

Figure 5. A) Simulated diffraction efficiency for order 0 (blue), 1 (green), 2 (red), 3 (black) and experimental dots as a function of time with diffusion in depth. B) Experimental measurements with PVA/AA. 
To validate the usefulness of the model in predicting the behavior of different photopolymers without cover plating, we introduced the parameters obtained from these photopolymers using zero frequency analysis in the diffusion model. The parameters introduced in the model were: $D=1.210^{-9} \mathrm{~cm}^{2} / \mathrm{s} ; \mathrm{k}_{\mathrm{R}}=0.0032 \mathrm{~cm}^{2} /(\mathrm{s} \cdot \mathrm{mW})$ [20], $\mathrm{Sh}=1.7 \mu \mathrm{m}$ [20], $\alpha=0.02$ $\mu \mathrm{m}^{-1}[20], \mathrm{M}_{0}=0.15, \mathrm{I}=1.1 \mathrm{~mW} / \mathrm{cm}^{2}, \gamma=1$ and $\mathrm{d}=100 \mu \mathrm{m}$. The simulation for the first 4 orders is presented in Figure 6 with the experimental results. The good agreement between the model and the experiments confirms the importance of directly determining parameters at the zero spatial frequency limit and also confirms the valeu of monomer diffusion in Biophotopol.

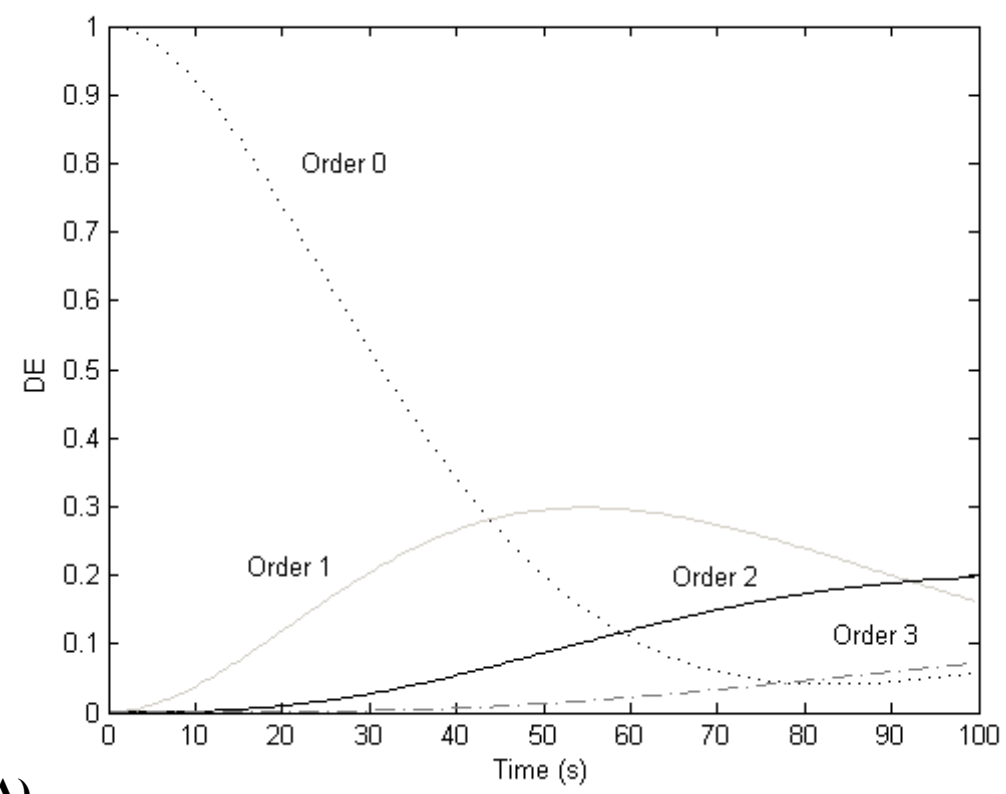

A) 


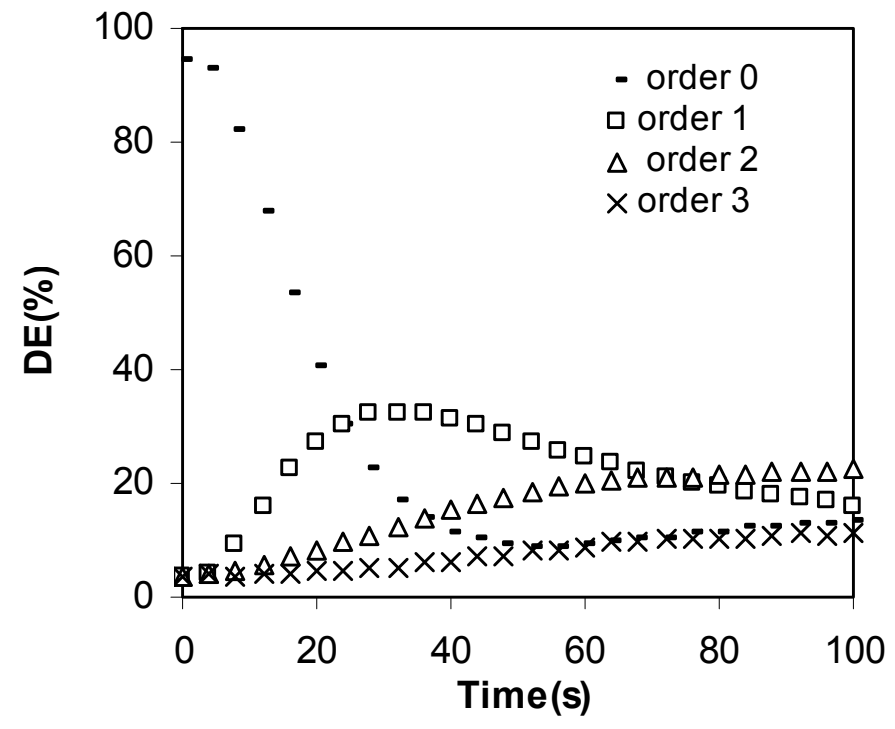

Fig. 6. A) Simulated diffraction efficiency for order $0,1,2,3$ and experimental dots as a function of time with diffusion in depth. B) Experimental measurements with PVA/NaAO.

\section{CONCLUSIONS AND PERSPECTIVES}

Using a spatial zero frequency analysis and very low spatial frequency recording, we characterized four different photopolymers with and without cover plating. The zero spatial frequency analysis allows the polymerization rate, shrinkage, relation between intensity and polymerization, etc to be directly measured. We have demonstrated that PVA/AA and PVA/NaAO materials can be made with similar polymerization rates and phase shifts. In addition, in our comparison of materials with cover plating, we have shown that the index variation is slightly higher in PEA photopolymers with liquid crystal molecules than in the Bayfol photopolymer. Nevertheless, the stability and conservation is clearly better in the Bayfol material. With this material, we recorded gratings with periods of $168 \mu \mathrm{m}$ and $336 \mu \mathrm{m}$, and the diffraction efficiency of the different orders was lower than $1 \%$. In addition, we recorded diffraction gratings using PVA/AA and PVA/NaAO materials with good results. Using a surface relief model we reproduced the exact behavior of the main diffracted orders for short and long exposures. This confirms that monomer diffusion is around $1.510^{-8} \mathrm{~cm}^{2} / \mathrm{s}$ in PVA/AA, and $1.210^{-9} \mathrm{~cm}^{2} / \mathrm{s}$ in PVA/NaAO.

\section{Acknowledgments}

This work was supported by the Ministerio de Ciencia e Innovación of Spain under projects FIS2008-05856-C02-01 and FIS2008-05856-C02-02 and by the Generalitat Valenciana of Spain under project PROMETEO/2011/021

\section{REFERENCES}

[1] Weiser, M.-S., Bruder, F.-K., Fäcke, T., Hönel, D., Jurbergs, D. and Rölle, T. (2010), Self-Processing, DiffusionBased Photopolymers for Holographic Applications. Macromolecular Symposia, 296: 133-137 (2010). 
[2] Y. Tomita, K. Furushima, K. Ochi, K. Ishizu, A. Tanaka, M. Ozawa, M. Hidaka, and K. Chikama, "Organic nanoparticle (hyperbranched polymer)-dispersed photopolymers for volume holographic storage," Appl. Phys. Lett. 88, 071103 (2006).

[3] J. Ashley, M.-P. Bernal, G. W. Burr, H. Coufal, H. Guenther, J. A. Hoffnagle, C. M. Jefferson, B. Marcus, R. M. MacFarlane, R. M. Shelby, G. T. Sincerbox, "Holographic Data Storage Technology,” IBM J. Res. Develop. 44, 3, 341$368,(2000)$.

[4] G. P. Nordinand, A. R. Tanguay, Jr., "Photopolymer-based stratified volume holographic optical elements," Opt. Lett. 17, 1709-1711, (1992).

[5] F. T. O’Neill, A. J. Carr, S. M. Daniels, M. R. Gleeson, J. V. Kelly, J. R. Lawrence, J. T. Sheridan, "Refractive elements produced in photopolymer layers," J. Mat. Sci. 40, 15, 4129-4132, (2005).

[6] J. Zhang, K. Kasala, A. Rewari, K. Saravanamuttu, "Self-trapping of spatially and temporally incoherent white light in a photochemical medium," J. Am. Chem. Soc. 128, 406-407, (2006).

[7] A. C. Sullivan, M. W. Grabowski, R. R. McLeod, "Three-dimensional direct-write lithography into photopolymer," Appl. Opt. 46, 3, 295-301, (2007).

[8] R. K Kostuk, J. Castro, D. Zhang "Holographic low concentration ratio solar concentrators," in Frontiers in Optics, OSA Technical Digest (CD) (Optical Society of America, 2009), paper FMB3.

[9] A. Márquez, S. Gallego, M. Ortuño, E. Fernández, M. L. Álvarez, A. Beléndez, I. Pascual, "Generation of diffractive optical elements onto a photopolymer using a liquid crystal display," Proc. SPIE 7717 (2010).

[10] S. Gallego, M.. Ortuño, C. Neipp, E. Fernández, A. Beléndez, and I. Pascual, "Improved maximum uniformity and capacity of multiple holograms recorded in absorbent photopolymers," Opt. Express 15, 9308-9319 (2007)

[11] L. M. C. Sagis, "Generalised curvature expansion for the surface internal energy," Physica A 246, 3, 591-608, (1997).

[12] S. Abe, J. T. Sheridan, "Curvature correction model of droplet profiles," Phys. Lett. A 253, 317-321, (1999).

[13] C. E. Close, M. R. Gleeson and J. T. Sheridan, "Monomer Diffusion Rates in Photopolymer Material: Part I: Low Spatial Frequency Holographic Gratings" JOSA B 28, 658-666, (2011).

[14] S. Gallego, A. Márquez, M. Ortuño, S. Marini, J. Francés "High environmental compatibility photopolymers compared to PVA/AA 3 based materials at zero spatial frequency limit" Opt. Materials 33, 531-537. (2010).

[15] S. Gallego, A. Márquez, S. Marini, E.a Fernández, M. Ortuño, and I. Pascual, "In dark analysis of PVA/AA materials at very low spatial frequencies: phase modulation evolution and diffusion estimation," Opt. Express 17, 1827918291 (2009)

[16] K. Pavani, I. Naydenova, S. Martin, and V. Toal, "Photoinduced surface relief studies in an acrylamide-based photopolymer," J. Opt. A, Pure Appl. Opt. 9, 43-48 (2007).

[17] I. Naydenova, E. Mihaylova, S. Martin, and V. Toal, "Holographic patterning of acrylamide-based photopolymer surface," Opt. Express 13, 4878-4889 (2005).

[18] S. Gallego, A. Márquez, M. Ortuño, S. Marini, I. Pascual and A. Beléndez "Monomer diffusion in sustainable photopolymers for diffractive optics applications" Opt. Materials, 33, 1626-1629 (2011).

[19] K. Hashimoto, W.N. Aldridge, Biochemical studies on acrylamide, a neurotoxic 535 agent, Biochem. Pharmacol. 19 2591-2604 (1970).

[20] S. Gallego, A. Márquez, M. Ortuño, J. Francés, S. Marini, A. Beléndez, and I. Pascual, "Surface relief model for photopolymers without cover plating," Opt. Express 19, 10896-10906 (2011) 